\title{
KULTURELLE DEUTUNGSMUSTER INTERKULTURELLEN HANDELNS. BEDEUTUNG FÜR DIE GRUNDLAGENFORSCHUNG UND ANWENDUNG IM DAF-BEREICH ${ }^{1}$
}

\author{
BARBARA HEINSCH (OVIEDO)
}

\begin{abstract}
CULTURAL INTERPRETATION PATTERNS OF INTERCULTURAL ACTION. RELEVANCE FOR BASIC RESEARCH AND APPLICATION IN THE FIELD OF GERMAN AS A FOREIGN LANGUAGE
\end{abstract}

This contribution analyzes the cultural interpretation patterns of intercultural action. It focuses first of all on the revision of the European Union's language policy and the concept of intercultural competence used in European regulations. Then the diversity of research approaches is highlighted. The article argues for a redefinition of cultural interpretation patterns. In order to exemplify how a researcher and even study participants themselves can fall into the trap of stereotypes, the design of a survey in the field of German as a Foreign Language among students with different mother tongues is presented and the results regarding cultural items are discussed.

Key words: educational standards, intercultural competence, intercultural action, stereotypes, field research

\section{ABSTRAKT \\ INTERPRETAČNÍ VZORCE MEZIKULTURNÍHO JEDNÁNÍ. VÝZNAM PRO ZÁKLADNÍ VÝZKUM A APLIKACI V OBLASTI NĚMČINY JAKO CIZÍHO JAZYKA}

Př́spěvek analyzuje kulturní interpretační modely mezikulturního jednání. Zaměřuje se především na revizi jazykové politiky Evropské unie a konceptu interkulturní kompetence používaného $\mathrm{v}$ evropských předpisech. Dále je zdůrazněna rozmanitost dnes uplatňovaných výzkumných př́stupů. Článek argumentuje ve prospěch nového vymezení kulturních interpretačních vzorců. Abychom mohli sledovat, jak se výzkumník a dokonce i samotní účastníci studia dostávají do pasti stereotypů, je prezentován návrh průzkumu v oblasti němčiny jako cizího jazyka mezi studenty s různými mateřskými jazyky a jsou diskutovány první výsledky jeho aplikace.

Klíčová slova: němčina jako cizí jazyk, mezikulturní jednání, Evropská unie, jazyková politika.

1 Dieser Beitrag wurde im Rahmen des von der Universität Oviedo unterstützten Forschungsprojektes ALCE verfasst (Referenz: SV-16-GRUPUO-ALCE). 


\section{Kontextualisierung: Zielsetzung der europäischen Sprachenpolitik}

Spätestens seit der Ausrufung des Europäischen Jahres der Sprachen 2001 bemüht sich die Europäische Union um eine weitgreifende Sprachenpolitik, die Mehrsprachigkeit mit sozio-ökonomischem Mehrwert verbindet und von daher für die Erhöhung der Effizienz und der Qualität des Sprachenlernens einerseits und für Messbarkeit von Sprachenkompetenz andererseits Grundsteine setzt. Mehrsprachigkeit wird als Voraussetzung für interkulturelle Kommunikationskompetenzen verstanden, die interkulturelle Kompetenz an sich aber überraschend wenig thematisiert. Trotzdem werden seit der Jahrhundertwende praktisch jedes Jahr Dokumente der Europäischen Union publiziert, die das Sprachenlernen, Mehrsprachigkeit und interkulturelle Verständigung in Europa in den Blick nehmen und Initiativen zu deren Umsetzung vorschlagen. In dem folgenden Abriss sollen die wichtigsten erwähnt werden, da sie die Forschungslandschaft und den Spielraum der Bildungsinstitutionen mitgestalten und sich umgekehrt die Defizite in der Erforschung der interkulturellen Kompetenz in ihnen widerspiegeln.

Die Lissabon-Strategie erfasst Fremdsprachen als eine der neuen, durch lebenslanges Lernen (life long learning $L L L$ ) zu vermittelnden Grundfertigkeiten (vgl. Europäischer Rat 2000: Punkt 26). In demselben Jahr wird der Entschluss gefasst, 2001 als Europäisches Jahr der Sprachen auszurufen, um die EU-Bürger, „durch Sensibilisierungs- und Aufklärungsmaßnahmen, zum Erlernen mehrerer Fremdsprachen anzuregen“ (Europäisches Parlament 2000). Einen wesentlichen Schritt zur Umsetzung der europäischen Sprachenpolitik stellt der Gemeinsame Europäische Referenzrahmen für Sprachen dar (Europarat 2001). Jedoch wird der Begriff der interkulturellen Kompetenz überraschenderweise dort nicht behandelt. Geneviève Zarate erläutert dazu, dass in einer ersten Version des Referenzrahmens von 1996 Bezugnahmen auf den Anderen - „the relation to the other" - und Nuancierungen zum Begriff „intercultural awareness“ existierten, die aber in der definitiven Ausgabe auf Grund von Schwammigkeiten in der Definition verschwanden (Zarate 2003: 98-100, hier 99 u. 100). Unter dem Titel Developing the intercultural dimension in language teaching von Byram/Gribkova/Starkey erscheint 2002 unter der Herausgeberschaft des Europarats eine praktische Einführung für Lehrkräfte. Dieselbe Dimension wird auch 2003 in Intercultural Competence, herausgegeben von Michael Byram, besprochen. Die Autoren fokussieren sich hier nicht nur auf das Fremdsprachenlernen und -lehren, sondern auch auf soziokulturelle Bezüge, Identitäten und Mehrsprachigkeit, die als Vorbedingung für interkulturelle Kompetenz anzusehen sind (vgl. Byram 2003). Gleichzeitig sieht die Europäische Kommission einen Aktionsplan für 2004-2006 zur Förderung des Sprachenlernens und der Sprachenvielfalt vor, und im August 2005 veröffentlicht sie den Europäischen Indikator für Sprachenkompetenz. Es handelt sich um eine Mitteilung, in der „Parameter und Durchführungsmodalitäten zur Umsetzung des Indikators“ (Kommission 2006) vorgeschlagen, aber auch Schwierigkeiten bekannt werden:

Es liegt also noch ein längerer Weg vor uns, bis jeder Bürger über die Sprachkenntnisse und die interkulturelle Kompetenz verfügt, um das Potenzial der Unionsbürgerschaft voll ausschöpfen zu können, und bis die Mitgliedstaaten die Vorgabe der Staats- und Regie- 
rungschefs erfüllt haben, dass alle Schüler vom jüngsten Kindesalter an mindestens zwei Fremdsprachen erlernen. (Kommission 2005a: 5)

Der Indikator soll aber auch „zuverlässige Daten zu den tatsächlichen Fremdsprachenkenntnissen junger Menschen und wertvolle Informationen für Entscheidungsträger/-innen liefern." (Kommission 2005b: 7) Im November 2005 dehnt die Kommission mit Einer neuen Rahmenstrategie für Mehrsprachigkeit ihre Bemühungen auf die Forschung im Bereich der Sprachenvielfalt aus, jedoch nicht um ihrer selbst willen, denn: „Für globale Marketing- und Verkaufsstrategien gewinnen interkulturelle Kommunikationskompetenzen zunehmend an Bedeutung." (Kommission 2005b: 9) Das Jahr 2008 scheint ganz im Zeichen der Mehrsprachigkeit und des interkulturellen Dialogs zu stehen: Die Europäische Kommission veröffentlicht die Vorschläge der von ihr eingesetzten Intellektuellengruppe für den interkulturellen Dialog, die 2007 in drei Sitzungen zusammenkam und deren Diskussionsergebnisse in dem Dokument Eine lohnende Herausforderung. Wie die Mehrsprachigkeit zur Konsolidierung Europas beitragen kann (vgl. Intellektuellengruppe 2008) zusammengetragen wurden. Diese Gruppe hat den Ausdruck „persönliche Adoptivsprache" geprägt, die keineswegs als Fremdsprache, sondern gewissermaßen als zweite Muttersprache zu verstehen ist und sich klar von einer internationalen Verkehrssprache unterscheide (vgl. Intellektuellengruppe 2008: 11). Die letztere

würde von den Anforderungen der Kommunikation im weiteren Sinne diktiert, die andere von einem Bündel persönlicher Beweggründe geleitet, die mit dem individuellen oder familiären Lebensweg, emotionalen Bindungen, beruflichen Neigungen, kulturellen Präferenzen, intellektueller Neugier usw. zusammenhängen. (Intellektuellengruppe 2008: 12)

Im Mai 2008 erscheinen das Weißbuch zum interkulturellen Dialog und die Schlussfolgerungen des Rates vom 22. Mai 2008 zu interkulturellen Kompetenzen, in denen die Rolle des Sprachenlernens und der Übersetzung für den Erwerb interkultureller Kompetenzen anerkannt wird (vgl. Rat der Europäischen Union 2008b: C141/15); weiterhin die Mitteilung Mehrsprachigkeit: Trumpfkarte Europas, aber auch gemeinsame Verpflichtung, die ausdrücklich die Menschen „und ihre Fähigkeit, sich mehrerer Sprachen zu bedienen [...] und als aktive Bürger am gesellschaftlichen Leben teilzunehmen " (Kommission 2008: 5) ins Zentrum rückt und in der es darum geht, „das Bewusstsein für den Wert der Sprachenvielfalt in der EU und für die von dieser Vielfalt ausgehenden Chancen zu schärfen und den Abbau von Hindernissen für den interkulturellen Dialog zu fördern." (Kommission 2008: 5) Die Entschliessung des Rates vom 21. November 2008 zu einer europäischen Strategie für Mehrsprachigkeit bekräftigt die vorangegangenen Initiativen. Zur Förderung des interkulturellen Dialogs soll verstärkt die Übersetzertätigkeit unterstützt werden (Rat der Europäischen Union 2008a: 3), womit ausschließlich die institutionelle Ebene angesprochen wird. Was der Begriff aber für den einzelnen Bürger zu bedeuten hat und wie dieser Interkulturalität leben kann, bleibt offen. Mit der Autobiography of intercultural encounters schließlich liegt ein von Michael Byram u.a. ausgearbeitetes Projekt vor, das - neben einer aufschlussreichen Einführung - Hilfestellungen dazu bietet, wie man mit tagebuchartigen Eintragungen in der Praxis ganz individuell die Vorbedingungen für eine kulturelle Offenheit schaffen kann, die zu einem interkulturellen Dialog 
führen sollte (vgl. Byram 2009: 9-11). Dieser Ansatz stellt in sich möglicherweise eine Resignation dar, denn auf Grund der Diskrepanz zwischen den „bildungspolitischen Zielen und der tatsächlichen curricularen Ausgestaltung interkultureller Kompetenz “ (Eberhardt 2013: 60; vgl. Rodríguez/Heinsch 2013: 230-238) sind die interkulturellen Bemühungen größtenteils dem Einzelnen selbst überlassen. Von daher habe der

Europarat jedenfalls und allen voran Michael Byram [...] sich nach anfänglicher Hoffnung in den letzten Jahren dezidiert auf Formen der autobiographischen Selbsteinschätzung (im Gegensatz zur Fremdevaluation) von interkulturellen Erfahrungen in ihrer Bedeutsamkeit und in ihren Auswirkungen durch die Betroffenen beschränkt. (Vollmer 2010: 52f.)

Tatsächlich scheint die Europäische Kommission sprachliche Kompetenzen endgültig mit ökonomischem Mehrwert zu verknüpfen, wie aus neueren Publikationen ersichtlich wird: In Language competences for employability, mobility and growth lautet eine Kapitelüberschrift: „Language skills are a ,must' for the modern economy..." (Europäische Kommission 2012: 4), und die Schlussfolgerungen des Rates vom 20. Mai 2014 zur Mehrsprachigkeit und zur Entwicklung von Sprachenkompetenz zielen auf höhere Effizienz und Qualität des Sprachenlernens und des Sprachunterrichts ab (vgl. Rat der Europäischen Union 2014: C 183/27).

Zusammenfassend kann festgestellt werden, dass die Behandlung der interkulturellen (Kommunikations-)Kompetenz faktisch an Fremdsprachendidaktiker und -experten wie beispielsweise Michel Byram, Geneviève Zarate und Gerhard Neuner delegiert wird, deren Arbeiten zur interkulturellen Dimension des Sprachenlernens bahnbrechend waren, die aber auch feststellen, dass die Debatte um sie über die rein politische nicht hinausgegangen sei; ein tiefgehender Ansatz bedürfe neuer Parameter im Bereich des Fremdsprachenlehrens und -lernens (z.B. die der Globalisierung, der eigenen Erfahrung, der Interaktionen zwischen europäischen und nichteuropäischen Sprachen, des Beitrags der Sprachen zur Objektivierung der Identitätskriterien), deren Komplexität die Reflexion der Institutionen bisher jedoch eher lähme (Zarate 2010: 16f.).

\section{Forschungsansätze im Spannungsverhältnis}

An die Erkenntnis, dass das Erlernen von Fremdsprachen der Entwicklung einer interkulturellen Handlungsfähigkeit per se zuträglich ist (vgl. Europarat 2001: 51), knüpfen die meisten Dokumente der Union an, wenn sie den Beitrag der Mehrsprachigkeit zum interkulturellen Dialog hervorheben, auch wenn sie die Entwicklung fremdsprachlicher und interkultureller Kompetenzen vorrangig mit Wettbewerbsvorteilen, sprich mit Wohlstand in Verbindung setzen (vgl. Kommission der Europäischen Gemeinschaften 2008: 8f.). Das tatsächlich erreichte sprachliche Kompetenzniveau vieler Europäer wird im Allgemeinen jedoch als noch unzureichend erachtet (vgl. Europäische Kommission 2012: 7-9).

Ein Mangel an klaren Niveauvorgaben für interkulturelle Kompetenzen durchzieht nicht nur die Bildungsstandards der Union. Auch in der geisteswissenschaftlichen Forschung herrscht bis weit in die Nuller Jahre und teilweise darüber hinaus Uneinigkeit 
bezüglich der Begriffe Interkulturalität, interkulturelle Kompetenz oder interkulturelles Lernen. Laut Rathje bewegen sich

[d]ie Stellungnahmen zu Zielvorstellungen interkultureller Kompetenz [...] zwischen eher ökonomisch orientierten Konzepten, die vor allem Effizienzgesichtspunkte in den Vordergrund stellen und eher geisteswissenschaftlichen, bzw. erziehungswissenschaftlichen Ansätzen, die Effizienzerwägungen gegenüber skeptisch eingestellt sind und vor allem den Aspekt menschlicher Weiterentwicklung in der interkulturellen Interaktion betonen. (Rathje 2006: 4)

Darüber hinaus stellt Heiser fest, dass „viele interkulturelle Ansätze von einer argumentatorischen Leerstelle“ (Heiser 2013: 243) zeugen und „ein wenig reflektierter Lernbegriff psychologisch-kognitivistischer Abstammung“ (Heiser 2013: 247) vorherrsche. Interkulturelles Lernen würde „als (Konstruktions)Prozess begriffen, der ergebnisorientiert auf das effizient-erfolgreiche Verhalten gegenüber Vertretern anderer Kulturen abzielt." (Heiser 2013: 247) Desweiteren betonen alle auf dem Kontrastpaar Fremdes /Eigenes fußenden Ansätze im Grunde genommen wieder die Differenz, die es eigentlich $\mathrm{zu}$ integrieren gilt. Sie gehen von einem geschlossenen Kulturbegriff aus, der deterministisch festschreibt, was eine bestimmte Gruppe von Menschen (z.B. einer Nationalität) charakterisiert und sie zusammenhält und wie sie in bestimmten Fällen reagiert oder zu reagieren hat. Dieser geschlossene Kulturbegriff liegt laut Conti selbst dem Weißbuch zum Interkulturellen Dialog zu Grunde, da „statische, homogene soziale Gruppen angenommen [werden], obwohl es sich um Konstrukte handelt, die durch innere Differenz gekennzeichnet sind.“ (Conti 2012: 303) Auch „das unzulässig vereinfachende Verständnis von Individuen als einer Nationalität zugehörig gerät vor allem in einer Zeit von fortgeschrittener Globalisierung an seine Grenzen." (Conti 2012: 303) Geht nicht auch der Begriff der interkulturellen Kompetenz, der als die Fähigkeit beschrieben wird, eine „kulturelle Zwischenposition“ (Wierlacher 2003: 260), eine gemeinsame Mitte, eine neue Ordnung zu stiften (vgl. Wierlacher 2003: 262), von festgelegten Kategorien aus - eben weil diese auf Unterscheidbarkeit beruhen -, besonders wenn Andersartigkeit immer nur auf kulturelle Elemente zurückgeführt wird?

Conti, die für einen offenen Kulturbegriff plädiert, stellt sich die Frage, wann Andersartigkeit - „die in jeder menschlichen Begegnung und sogar innerhalb des eigenen Selbst wiederzufinden ist“" (Conti 2012: 304) - zur kulturellen Unterscheidung herangezogen und eine dialogische Interaktion als interkulturell bezeichnet werden könne. Sie definiert dazu drei Fälle, von denen der erste und dritte - bei denen das Gegenüber mit einem bestimmten Kollektiv in Verbindung gebracht werde - Stereotype und Vorurteile hervorrufe (vgl. Conti 2012: 304). An dieser Stelle soll auf den Terminus „kulturelle Deutungsmuster“ Bezug genommen werden, der als Voraussetzung einer Eingrenzung dessen bedarf, was mit Kultur gemeint ist. In der Kulturwissenschaft wurde sie als „lebensweltliches Hintergrundwissen definiert“ (Altmayer 2004: 147), das möglicherweise für verschiedene Gruppen sehr heterogen ausfallen kann. Wie kann aber angenommen werden, „national oder ethnisch definierte Gruppen seien nach innen mehr oder weniger homogen und ließen sich von anderen Gruppen mehr oder weniger klar abgrenzen“ (Altmayer 2004: 149), zumal eine soziale Zugehörigkeit auch immer subjektiv von dem 
Individuum selbst bestimmt wird und bestimmt werden muss? Hieße das nicht, „in die Fallen des homogenisierenden Kulturbegriffs zu tappen“ (Altmayer 2004: 147)? Kulturelle Deutungsmuster, die als eine Art Vorprogrammierung des Denkens, Fühlens und Handelns, welche das Individuum mit anderen seiner Gruppe Zugehörigen teilt, werden aktiviert, indem von dem gemeinsamen - unterstellten - Wissen Gebrauch gemacht wird. Als Gruppen identifiziert werden können Kommunikationsgemeinschaften, ein von mehreren Forschern adoptierter Begriff, der die Großgruppen realitätsgerechter auffächert. Dabei können diese dieselbe Muttersprache teilen oder auch nicht. Das Postulat eines auf einem dynamisch-flexiblen Kulturbegriff gegründeten interkulturellen Kompetenzmodells (vgl. Eberhardt 2013: 70) kann jedoch nicht darüber hinwegtäuschen, dass kulturelle Deutungsmuster als „,intersubjetive Wissensstrukturen“ (Altmayer 2004: 154) einen gewissen Normcharakter beibehalten und der von vornherein auch berücksichtigt wird, denn sonst würde jegliche Form von Interkulturalität ad absurdum geführt. Eine Chance, kulturelle Deutung nicht von vornherein festzuschreiben, besteht in der „Epoché - der temporären Urteilsenthaltung“ (Heiser 2013: 315), die bei diesem Autor auch gleichzeitig als interkulturelles Lernziel gesetzt wird, das bildungstheoretisch nur durch „Habitualisierung der Überlegung“ erreicht werden kann (Heiser 2013: 372), d.h.:

Bei einer Interkulturellen Didaktik kann es sich also nur um ein vorbereitendes sowie nachbereitendes Lehren handeln, welches einerseits propädeutisch auf das Vorwissen und die Vorurteile in Hinsicht auf ein späteres Agieren im Zwischen Bezug nimmt und andererseits die bereits gemachten Erfahrungen und den Umlernvollzug epilogisch thematisiert. (Heiser 2013: 360)

Zusammenfassend gesagt, geht es wesentlich darum, Stereotypen, die im interkulturellen Handeln überwunden werden sollen, nicht durch neue, im Namen eben dieser Interkulturalität, für die es ja per definitionem verschiedene Kulturen geben muss, festzuschreiben. Paradoxerweise bleibt aber auch die Forschung nicht vor dieser Gefahr gefeit, denn

on the one hand, the researchers defend and put forward the changeability and unstable nature of cultures, identities, "subjects" (i.e. the diverse diversities of each and every one of us), but on the other hand, through e.g. their corpus analyses, which resort to quantification or "soft" discourse analysis or contents analysis, they categorise study participants into national, religious, ethnic groups - and this limits the co-constructive aspects. (Dervin 2010: 4f.)

Das Plädoyer für eine Kursänderung in der Erforschung des interkulturellen Diskurses, indem Forscher zuallerst selbst ihren Ethnozentrismus, ihre Verallgemeinerungen und Stereotypen ablegen (vgl. Dervin 2011: 49), wird auch gleichermaßen für die Fremdsprachenlehre akut, wenn es heißt, interkulturelle Inhalte zu vermitteln und interkulturelle Kompetenzen zu bewerten, die dennoch nicht an neue Klischees gebunden sind. Dazu kann auch der Kulturbegriff nicht unhinterfragt bleiben, auch wenn er noch so sehr in den alltäglichen interkulturellen Begegnungen, transnationalen Politikszenarien und in den Medien gebraucht wird (vgl. Dervin 2011: 39): ,interculturality is understood as the positioning and negotiation of individuals who come from different spaces-times 
(rather than 'cultures' [...]). Identification but also the concept of intersubjectivity are thus central." (Dervin 2011: 38)

Fragt man nun abschließend nach interkultureller Bildung im Handlungsfeld Hochschule und Universität, so betreffen die Unklarheiten nicht nur die Fachebene, sondern auch die Akteure und Entscheidungsträger selbst. Sie hängt davon ab oder wird mitbestimmt davon, wie diese Akteure interkulturelle Bildung verstehen und welche Zielsetzungen sie verfolgen und letztendlich von ihrer eigenen interkulturellen Kompetenz (vgl. Weidemann/Nothnagel 2010: 126). Im folgenden Abschnitt, der sich mit einer Umfrage unter Studierenden beschäftigt, sollen nicht nur einige der Ergebnisse derselben aufgezeigt, sondern anhand kritischer Fragen auch die Deutungsmuster und kulturelle Verhaltensweisen von Dozierenden und Forschern beleuchtet werden, die sich auf ihre Studien auswirken können, z.B. durch die Ausarbeitung von Fragebögen und die Interpretation der Resultate.

\section{Die Behandlung einer Umfrage unter DaF-Studierenden diverser Muttersprachen}

Das Ziel der von Juni bis Juli 2016 an der Universität Münster im Bereich Deutsch als Fremdsprache durchgeführten Umfrage war nicht nur, kulturelle Lernmuster und interkulturelle Handlungsfähigkeit der Lernenden zu beobachten und diese mit Herkunftssprachen und kulturellen Herkunftskreisen in Beziehung zu setzen, sondern auch die Rolle der Forscherin, ihrer Deutungsmuster und ihren Einfluss auf die Ergebnisse der Studie zu untersuchen, denn sie/er kann einerseits die Fragen gezielt und voreingenommen aus eigenen Forscherinteressen und vor dem eigenen Kultur- und Erfahrungshorizont stellen, und andererseits Aussagen der Studienteilnehmer/-innen ohne Reflexion einfach übernehmen und sie als Beweismaterial benutzen, und dabei auch Gefahr laufen, sich selbst als impliziten Teilnehmer aus diesem Forschungsprozess auszublenden, obwohl durch ihn/sie die Daten und darauffolgenden Diskurse erst zustande kommen; das heißt also, er/sie kann in die Falle des „Othering“ (Dervin 2011: 39) tappen, was bedeutet, aus dem Anderen einen Anderen, einen Fremden zu machen. Von daher geht es in den folgenden Abschnitten dieses Beitrags nicht um die Auswertung der Umfrage selbst, sondern um die Beleuchtung des Vorgehens und der ihm zu Grunde liegenden Deutungsmuster. Dazu werden einige signifikante Beispiele ausgewählt, an denen die oben besprochenen Punkte veranschaulicht werden. Letztere dienen deshalb als Leitfaden für die weitere Untersuchung: 1. Findet die in den offiziellen Dokumenten vertretene Auffassung von Sprachen und Mehrsprachigkeit ihren Niederschlag in der Umfrage? 2. Werden die Niveaustufen zur Kompetenzerfassung berücksichtigt? 3. Wird von einer Verbindung zwischen Sprache, Kultur und Lern- und Lehrmustern ausgegangen? 4. Liegt der Ausarbeitung der Umfrage die Perspektive von dem Kontrastpaar Fremdes/Eigenes zu Grunde? 5. Wird von einem bestimmten Kulturbegriff ausgegangen? 6. Findet irgendeine Art von Stereotypierung statt, sowohl im Vorfeld als auch bei der Datenanalyse? 


\subsection{Prämissen}

Das Erhebungsinstrument bestand aus einem Fragebogen mit geschlossenen Fragen zu persönlichen Daten wie Geschlecht, Alter, Muttersprache(n), im Familienkreis gesprochene Sprache(n), Sprachkenntnisse (Niveau), dann folgten sieben offene Fragen. Insgesamt nahmen 122 Studierende teil, davon 19 in einer studienvorbereitenden Gruppe (im Alter zwischen 17 und 31 Jahren), der Rest in studienbegleitenden Gruppen (die meisten im Alter zwischen 20 und 25 Jahren). Im Falle der studienvorbereitenden Gruppe wurde der Fragebogen nach Expertenmeinung (Koordinatorin des Kurses) in einem vereinfachten Deutsch verfasst, da die Teilnehmer ein A-Niveau (GeRS-Skala) hatten, auch wenn einige sich etwas höher einschätzten. Es wurde ihnen jedoch - wie allen anderen Teilnehmern - die Möglichkeit geboten, in anderen Sprachen zu antworten (außer Deutsch auch auf Englisch, Französisch, Spanisch), damit sie von erhöhten Ausdrucksmöglichkeiten Gebrauch machen konnten. Den studienbegleitenden Gruppen wurden die Fragebögen außerdem in zweisprachiger Form (Deutsch/Englisch) vorgelegt.

Für die vorliegende Studie werden aus dem ganzen Fragenkomplex der Erhebung ${ }^{2}$ zwei (Frage zwei und sieben) vorgestellt, weil sie mit Kulturverständnis zu tun haben und Rückschlüsse auf Ansätze für ein interkulturelles Verständnis bieten, seitens der Studierenden und seitens der Forscherin, die die Antworten erfasst und interpretiert. Frage zwei: Sind die folgenden Aspekte beim Erlernen der deutschen Sprache für Sie eine Schwierigkeit? Warum? $\rightarrow$ e) Kultur (Version für die studienvorbereitende Gruppe: Sind die folgenden Aspekte beim Erlernen der deutschen Sprache für Sie schwierig? Warum? $\rightarrow$ e) Kultur). Frage sieben: Lehrstil. Welche Charakteristika sollte ein Deutschlehrer oder eine Deutschlehrerin besitzen und warum? (Version für die studienvorbereitende Gruppe: Lehrstil. Wie ist für Sie ein guter Deutschlehrer oder eine gute Deutschlehrerin?)

Der Formulierung der geschlossenen und offenen Fragen gingen folgende Überlegungen voran: Gibt es bestimmte Lernmuster für die verschiedenen Herkunftskulturen und von daher bestimmte Erwartungen an die Lehrpersonen? Werden sie durch eine Gruppierung um dieselbe Muttersprache herum festgeschrieben oder gar stereotypiert? Verstärkt eine homogene Gruppe solche eventuell existierenden Lernmuster oder bringt sie latente Tendenzen zu Tage? Werden Angehörige bestimmter Muttersprachen in Mischgruppen individueller und lösen sich mehr von kulturellen Gruppenzwängen, d.h. besinnen sie sich eher auf persönliche Eigenarten im Sprachenlernen und im Umgang mit Kommilitonen und der Lehrperson? Übertragen oder verstärken sich bei homogenen

\footnotetext{
Folgende Fragen wurden formuliert: 1. Warum lernen Sie Deutsch? 2. Sind die folgenden Aspekte beim Erlernen der deutschen Sprache für Sie eine Schwierigkeit? Warum? a) Aussprache und Betonung, b) Syntax, c) Grammatik, d) Wortschatz, e) Kultur 3. Lernstil. Welche Strategien haben Sie zum Deutschlernen oder für Fremdsprachen allgemein? 4. Arbeitsteilung im Klassenraum. Welche Arbeitsform (in der Gruppe, Partner- oder Einzelarbeit) fördert Ihren Lernprozess am meisten? Warum? 5. Materialien. Welche Materialien benutzen Sie am liebsten, um den Lernprozess zu optimieren? 6. Benutzen Sie das Web, um sich mit anderen in der Fremdsprache zu verständigen? Welchen Nutzen oder welche Vorteile hat das für Sie? Gibt es auch Nachteile? 7. Lehrstil. Welche Charakteristika sollte ein Deutschlehrer oder eine Deutschlehrerin besitzen und warum?
} 
Gruppen, d.h. mit derselben Muttersprache, bestimmte „Heimatschemata“ oder „heimatkulturelle“ Blickwinkel? Schmälert dies eventuell die Aufnahmebereitschaft für die neue Kultur und behindert es eine genuin interkulturell offene Handlungsweise?

\subsection{Diskussion der Leitfragen}

Insgesamt waren 32 verschiedene Muttersprachen vertreten; acht Teilnehmer gaben an zweisprachig zu sein, 114 einsprachig. Von den nach Muttersprachen geordneten Gruppen wurden zur näheren Untersuchung acht ausgewählt, weil sie höhere Teilnehmerzahlen vorweisen, die sich wie folgt verteilen: Arabischsprachige 17, Französischsprachige 17, Italienischsprachige 13, Spanischsprachige elf, Chinesischsprachige neun, Türkischsprachige sieben, Tschechischsprachige fünf und Japanischsprachige fünf. Mit dieser Einteilung und dem ersten hier vorgestellten Item der Umfrage, ob die (deutsche) Kultur Schwierigkeiten im Lernprozess bereite, soll auf die obigen Leitfragen eingegangen werden, und zwar speziell auf die erste, die fünfte und die sechste.

Zur ersten: Findet die in den offiziellen Dokumenten vertretene Auffassung von Sprachen und Mehrsprachigkeit ihren Niederschlag in der Umfrage? Ja, denn es wurde eine Auswahl an Sprachen zur Verfügung gestellt, in denen die Kommunikation zwischen Befragerin und Befragten stattfinden konnte, die auf dem Prinzip des gemeinsamen Nenners beruht, d.h. eines geteilten, und folglich auch mitgeteilten Wissens, und keineswegs auf Präferenzen oder Diskriminierung. Tatsächlich wurde von dieser Möglichkeit Gebrauch gemacht. Zur fünften: Wurde von einem bestimmten Kulturbegriff ausgegangen? Nein, was den Nachteil hat, dass die Befragungsteilnehmer nicht wussten, worauf diese Frage hinauslief und die entsprechenden Antworten oder Lücken nicht vergleichbar sind. Der Vorteil besteht jedoch darin, dass ein subjetiver oder intersubjektiver Kulturbegriff zum Ausdruck kommen konnte, wenn man Kultur als ein gemeinsames „lebensweltliches Hintergrundwissen“ (Altmayer 2004: 147) versteht, das Handeln und Fühlen mitbestimmt, aber nicht (vollständig) erklärt. Bei Beantwortung der Frage mit „ja“ wurden als Gründe beispielsweise folgende Aspekte zitiert: „As with every culture different to your own one, there are some cultural shocks. However, I don't find it as hard. I guess it also depends on how open-minded a person is. Nevertheless, I find that in Germany I have to be 'colder' than in Spain." (Spanischsprachige, B-Niveau); zweimal wurde die Anpassung an das Essen als schwierig empfunden (Französischsprachige und Türkischsprachiger, beide B-Niveau); die Ausdrucksweise des Deutschen sei oft zu direkt: „(z.B. Wollen wir uns mal treffen?) Für mich als Japanerin war das sehr schwer mich daran zu gewöhnen." (C-Niveau). Betrachtet man die Antworten auf die Frage nach Kultur der beiden muttersprachlich und zahlenmäßig homogenen Gruppen (die arabisch- und die französischsprachige), dann ergibt sich, dass $82,36 \%$ der Teilnehmer der ersten keinerlei Angaben machte, 11,77\% antworteten mit ja (sie hätten Schwierigkeiten wegen vieler verschiedener und schwieriger Wörter). Diese Ergebnisse können nicht nur damit in Verbindung gebracht werden, dass die Lerner Deutschkenntnisse auf A-Niveau hatten, denn bei der Frage nach dem Lehrstil drückten fast alle ihre Meinung aus. Möglicherweise fehlt es an anderen Kompetenzen oder hat paradoxerweise kulturelle oder politische Gründe, wenn man bedenkt, dass es sich bei der Gruppe um nichteuropäische Migranten handelte, die vielleicht eine kritische Antwort oder überhaupt eine für politisch nicht korrekt 
hielten. Hier ein kulturelles Deutungsmuster der Forscherin! Die französischsprachige Gruppe hingegen, die sich aus 13 Jurastudenten mit C-Niveau und vier weiteren Teilnehmern mit B-Niveau zusammensetzte, äußerte sich zu 82,36\%, keine Schwierigkeiten zu haben, 5,88\% hatten keine gute Erfahrung gemacht, wobei offen bleibt, inwiefern diese nicht auch persönlichen Umständen zuzuschreiben ist. Festzuhalten ist hier, dass die muttersprachlich homogenen Gruppen auf die Frage nach den Schwierigkeiten mit der Kultur mehrheitlich homogen antworteten. Zur sechsten Frage: Findet irgendeine Art von Stereotypierung statt, sowohl im Vorfeld als auch bei der Datenanalyse? Ja, wenn die Tatsache der Gruppierung der Teilnehmer/-innen um dieselbe Muttersprache als solche aufgefasst wird, weil mehr oder weniger homogene Äußerungen auf die ausgewählten Fragen erwartet oder vorausgesetzt werden; nein, wenn man bedenkt, dass jedweder Versuch von Systematisierung eine bestimmte Vorausschau auf die erwarteten Ergebnisse impliziert.

Anhand der Frage nach dem Lehrstil des Dozenten, d.h. wie man sich einen guten Deutschlehrer bzw. eine gute Deutschlehrerin vorstellt, sollen nun die restlichen Leitfragen diskutiert werden. Zur zweiten: Werden die Niveaustufen zur Kompetenzerfassung berücksichtigt? Ja, denn das Sprachniveau sollte schon im ersten Teil des Fragebogens angegeben werden; weiterhin wurden innerhalb der Muttersprachengruppen alle Antworten nach Sprachniveaus (A, B, C) klassifiziert und entsprechend ausgewertet. Abgesehen von einigen Gemeinsamkeiten - so z.B., dass der Dozent Geduld haben solle, was in fast allen Gruppen und auf den meisten Niveaus angeführt wurde - konnten auf B-Niveau Parallelen zwischen den französisch-, türkisch- und italienischsprachigen Studierenden festgestellt werden (der Fokus lag auf der Einstellung der Dozentin und auf relationalen Aspekten), und zwischen den spanisch- und chinesischsprachigen (es wurden eher kognitive Eigenschaften betont). Zur dritten Frage: Wird von einer Verbindung zwischen Sprache, Kultur und Lern- und Lehrmustern ausgegangen? Ja. Diese Verbindung wurde auch von mehreren Studienteilnehmer/-innen erkannt, besonders aus der französischsprachigen Gruppe der Jurastudenten (C-Niveau), und selbstbewusst zum Ausdruck gebracht: Der Dozent solle u.a. pädagogisch sein, die Studierenden verstehen („Irren ist menschlich.“) und sie nicht diskreditieren, wenn sie Schwierigkeiten haben, Mobilitätserfahrung in einem fremden Land und mit der Sprache haben, um auf die Bedürfnisse des Anderen auf Französisch eingehen zu können, ein oder zwei andere Sprachen beherrschen, Fragen beantworten und eine angemessene Menge an Arbeit ausführen. Dahingegen lässt sich an den Antworten der arabischsprachigen Anfängergruppe ein Lernverständnis ablesen, das wenig mit der Autonomie des Lerners und der Reflexion über den eigenen Lernprozess zu tun hat. Es erinnert an die traditionelle Rollenverteilung im Schulsystem, wobei der Wunsch, sie solle für sie mehr als eine Lehrerin sein, einen Hinweis auf das Bildungssystem der Herkunftskultur geben könnte: Sie solle glücklich und für die Studenten mehr sein als eine Lehrerin, alle Fragen beantworten, immer etwas Neues zeigen, manchmal härter durchgreifen, viel sprechen, viele Hausaufgaben geben (mehrmals genannt), im Kurs viel Kontakt herstellen, ab und zu Spaß machen. Zur vierten Frage: Liegt der Ausarbeitung der Umfrage die Perspektive von dem Kontrastpaar Fremdes/Eigenes zu Grunde? Nein. Aber bei der Behandlung des Lehrstils gehen verschiedene Teilnehmer explizit auf Kultur ein und kontrastieren die eigene („unsere Kultur") mit der deutschen, aber meist erst auf C-Niveau: Für die chinesischsprachige 
Gruppe ist wichtig, dass der Lehrer geduldig ist („weil die meisten von uns eine brandneue Sprache lernen", sonst gäben sie leicht auf, da sie die Lehrer nicht verärgern wollen). Er solle auch pünktlich und freundlich sein, das sei besonders für asiatische Schüler wichtig, da ihre Kultur ganz anders als die deutsche sei. Interessant ist auch folgende Reflexion: „Manchmal ist es für Einheimische schwer Grammatik zu erklären“, d.h. hier wird vom Dozenten implizit verlangt, dass er sich mit seiner eigenen Sprache auseinandersetzt und lernt, deren grammatischen Systeme Anderssprachigen zu erklären. Sich der eigenen Sprache bewusst zu werden scheint keine Selbstverständlichkeit zu sein. Aus der japanischsprachigen C-Gruppe kamen ähnliche Äußerungen: „Manche sagen >Ja, es ist einfach so.< Das versteht keiner und so kann man nicht lernen. "Die Kultur wurde ebenfalls zur Sprache gebracht. Man bräuchte nicht nur Sprachkenntnisse, sondern auch Verständnis für die deutsche Kultur. Alle Lerner haben unterschiedliche kulturelle Hintergründe und seien anders als Deutsche. Die Sprache könne man auch alleine lernen, aber die Kultur nicht. Es wird also ersichtlich, dass das Fremdsprachenniveau Einfluss auf den Umgang mit Kultur und auf den Blick auf Kultur hat.

\section{Fazit}

Die aufgezeigte Kluft zwischen bildungspolitischen Standards - besonders, was die Förderung und Evaluierung interkulturellen Handelns angeht - und fremdsprachendidaktischen Diskursen einerseits und Gegensätzlichkeit verschiedener Konzeptionen zu möglichen oder nicht möglichen interkulturellen Kompetenzmodellen andererseits macht deutlich, dass je nach Interessengemeinschaft Interkulturalität systematisiert und handhabbar gemacht werden soll, indem nationale oder kulturelle Vergleichskategorien fixiert und so paradoxerweise Stereotypen betont werden, oder auch für ein interkulturelles Kompetenzmodell plädiert wird, dass auf einem dynamisch-flexiblen Kulturbegriff basiert. Den meisten Ansätzen haftet jedoch ein Fehler an: Sie führen die Verschiedenheit des Anderen ausschließlich auf kulturelle Elemente zurück, so dass andere Gründe für ein bestimmtes Verhalten, z.B. psychologischer, persönlicher oder religiöser Art, nicht erkannt oder verzerrt wahrgenommen werden und das Konstrukt der Interkulturalität überdimensioniert erscheint. Die Forderung nach einem tragfähigen Strukturmodell interkulturellen Lernens und Handelns mag legitim sein, birgt aber immer die Gefahr einer - wenn auch ungewollten - erneuten Festschreibung kultureller Kategorien in sich, und nicht zuletzt durch Umbenennung, d.h. nichtkulturelle Umstände werden als kulturelle erfasst. 


\section{Anlage \\ Fragebogen für DaF-Lerner/-innen}

Bitte nehmen Sie sich ein bisschen Zeit, um diesen Fragebogen zu beantworten. Vielen Dank dafür! Gerne können Sie die Rückseite der einzelnen Blätter benutzen, wenn Sie mit dem Platz nicht auskommen. Sie können auf Deutsch, Englisch, Französisch oder Spanisch antworten.

Please answer this questionnaire. Thank you very much! You can answer in German, English, French or Spanish, and you can also write on the reverse if necessary.

Geschlecht / (man/ woman):

Alter / Age:

Muttersprache(n) / mother tongue(s):

Im Familienkreis gesprochene Sprachen / language(s) you speak in the family:

Sprachkenntnisse (Niveau) / language level in all languages you speak:

1. Warum lernen Sie Deutsch? / Why do you study German?

2. Sind die folgenden Aspekte beim Erlernen der deutschen Sprache für Sie eine Schwierigkeit? Warum? / Are the following aspects of German language difficult for you? Why?

a) Aussprache und Betonung / Pronunciation and accentuation

b) Syntax / syntax

c) Grammatik /grammar

d) Wortschatz / vocabulary

e) Kultur /culture

3. Lernstil. Welche Strategien haben Sie zum Deutschlernen oder für Fremdsprachen allgemein? / Learning style. How do you study German or any other foreign language? What strategies or methods do you use?

4. Arbeitsteilung im Klassenraum. Welche Arbeitsform (in der Gruppe, Partner- oder Einzelarbeit) fördert Ihren Lernprozess am meisten? Warum? / What working method in classroom helps you the most to optimize your learning process (team work, working with a partner or alone)? Why?

5. Materialien. Welche Materialien benutzen Sie am liebsten, um den Lernprozess zu optimieren? / What materials do you prefer to optimize your learning process?

6. Benutzen Sie das Web, um sich mit anderen in der Fremdsprache zu verständigen? Welchen Nutzen oder welche Vorteile hat das für Sie? Gibt es auch Nachteile? / Do you use the Web in order to communicate with others? Are there any benefits or advantages in doing so? Are there also disadvantages?

7. Lehrstil. Welche Charakteristika sollte ein Deutschlehrer oder eine Deutschlehrerin besitzen und warum? / Teaching style. How should a good German teacher be? Why? 


\section{LITERATUR}

Altmayer, Claus (2004): Kultur als Hypertext. Zu Theorie und Praxis der Kulturwissenschaft im Fach Deutsch als Fremdsprache. München: Iudicium.

Byram, Michael (Hg.) (2003): Intercultural Competence; online unter: https://rm.coe.int/16806ad2dd [Stand: 01.04.2017].

Byram, Michael / Gribkova, Bella / Starkey, Hugh (2002): Developing the intercultural dimension in language teaching: A practical introduction for teachers. Strasbourg: Council of Europe; online unter: http://give2all. org/pdf/dimension/5.pdf [Stand: 09.07.2018].

Byram, Michael u.a. (2009): Autobiography of intercultural encounters; online unter: http://www.coe.int/t/dg4 /autobiography/Source/AIE_en/AIE_context_concepts_and_theories_en.pdf [Stand: 30.03.2017].

Conti, Luisa (2012): Vom Realen ins Virtuelle und zurück: Wege des interkulturellen Dialogs. In: Reutner, Ursula (Hg.): Von der digitalen zur interkulturellen Revolution. Baden-Baden: Nomos, 293-316.

Dervin, Fred (2010): Assessing intercultural competence in Language Learning and Teaching: a critical review of current efforts. In: Dervin, Fred / Suomela-Salmi, Eija (Hgg.): New Approaches to Assessment in Higher Education. Bern: Peter Lang, 157-173; online unter: http://users.utu.fi/freder/Assessing\%20intercultural\%20 competence\%20in\%20Language\%20Learning\%20and\%20Teaching.pdf [Stand: 13.02.2018].

Dervin, Fred (2011): A plea for change in research on intercultural discourses: A liquid' approach to the study of the acculturation of Chinese students. In: Journal of Multicultural Discourses 6, H. 1, 37-52; online unter: http://www.tandfonline.com/doi/pdf/10.1080/17447143.2010.532218 [Stand: 05.04.2017].

Eberhardt, Jan-Oliver (2013): Interkulturelle Kompetenzen im Fremdsprachenunterricht. Auf dem Weg zu einem Kompetenzmodell für die Bildungsstandards. Trier: Wissenschaftlicher Verlag.

Europäische Kommission (2003): Förderung des Sprachenlernens und der Sprachenvielfalt: Aktionsplan 2004-2006; online unter: http://eur-lex.europa.eu/legal-content/DE/TXT/HTML/?uri=LEGIS SUM:c11068\&from=DE [Stand: 06.04.2017].

Europäische Kommission (2012): Language competences for employability, mobility and growth; online unter: http://eur-lex.europa.eu/legal-content/EN/TXT/PDF/?uri=CELEX:52012SC0372\&from=EN [Stand: 30.03.2017].

Europäischer Rat (2000): (Lissabon-Strategie). 23. und 24. März 2000 Lissabon. Schlussfolgerungen des Rates; online unter: http://www.europarl.europa.eu/summits/lis1_de.htm?textMode=on [Stand: 05.04.2017].

Europäisches Parlament und Rat der Europäischen Union (2000): Europäisches Jahr der Sprachen 2001; online unter: http://eur-lex.europa.eu/legal-content/DE/TXT/HTML/?uri=LEGISSUM:c11044\&from=DE [Stand: 06.04.2017].

Europarat (2001): Gemeinsamer europäischer Referenzrahmen für Sprachen: lernen, lehren, beurteilen. Berlin /München/Wien/Zürich/New York.

Europarat (2008): Weißbuch zum interkulturellen Dialog. Gleichberechtigt in Würde zusammenleben; online unter: http://www.coe.int/t/dg4/intercultural/Source/Pub_White_Paper/WhitePaper_ID_GermanVersion. pdf [Stand: 30.03.2017].

Heiser, Jan Christoph (2013): Interkulturelles Lernen. Eine pädagogische Grundlegung. Würzburg: Königshausen \& Neumann.

Intellektuellengruppe für den interkulturellen Dialog (2008): Eine lohnende Herausforderung. Wie die Mehrsprachigkeit zur Konsolidierung Europas beitragen kann; online unter: http://www.migration-online.de/data /euberichtzweitemuttersprache.pdf [Stand: 01.04.2017].

Kommission der Europäischen Gemeinschaften (2005a): Mitteilung der Kommission an das Europäische Parlament und den Rat. Europäischer Indikator für Sprachenkompetenz; online unter: http://eur-lex.europa.eu /legal-content/DE/TXT/PDF/?uri=CELEX:52005DC0356\&from=DE [Stand: 06.04.2017].

Kommission der Europäischen Gemeinschaften (2005b): Mitteilung der Kommission an den Rat, das Europäische Parlament, den Europäischen Wirtschafts- und Sozialausschuss und den Ausschuss der Regionen. Eine neue Rahmenstrategie für Mehrsprachigkeit; online unter: http://eur-lex.europa.eu/LexUriServ/LexUriServ. do?uri=COM:2005:0596:FIN:de:PDF [Stand: 05.04.2017].

Kommission der Europäischen Gemeinschaften (2006): EUR-Lex - c11083 - EN 18.01.2006; online unter: http://eur-lex.europa.eu/legal-content/DE/TXT/?uri=URISERV\%3Ac11083 [Stand: 07.04.2017]. 
Kommission der Europäischen Gemeinschaften (2008): Mitteilung der Kommission an den Rat, das Europäische Parlament, den Europäischen Wirtschafts- und Sozialausschuss und den Ausschuss der Regionen. Mehrsprachigkeit: Trumpfkarte Europas, aber auch gemeinsame Verpflichtung; online unter: http://eur-lex.europa.eu /legal-content/DE/TXT/PDF/?uri=CELEX:52008DC0566\&from=DE [Stand: 03.04.2017].

Rat der Europäischen Union (2008a): Entschliessung des Rates vom 21. November 2008 zu einer europäischen Strategie für Mehrsprachigkeit. In: Amtsblatt der Europäischen Union, 16.12.2008, C320/1-C320/3; online unter: http://eur-lex.europa.eu/legal-content/DE/TXT/PDF/?uri=CELEX:32008G1216(01)\&from=DE [Stand: 01.04.2017].

Rat der Europäischen Union (2008b): Schlussfolgerungen des Rates vom 22. Mai 2008 zu interkulturellen Kompetenzen. In: Amtsblatt der Europäischen Union, 07.06.2008, C141/14-C141/16; online unter: http://eur-lex. europa.eu/legal-content/DE/TXT/PDF/?uri=CELEX:52008XG0607(01)\&from=DE [Stand: 30.03.2017]

Rat der Europäischen Union (2014): Schlussfolgerungen des Rates vom 20. Mai 2014 zur Mehrsprachigkeit und zur Entwicklung von Sprachenkompetenz. In: Amtsblatt der Europäischen Union, 16.04.2014, C183/26-C183/29; online unter: http://eur-lex.europa.eu/legal-content/DE/TXT/PDF/?uri= CELEX:52014XG0614(06)\&from=DE [Stand: 01.04.2017].

Rathje, Stefanie (2006): Interkulturelle Kompetenz - Zustand und Zukunft eines umstrittenen Konzepts. In: Zeitschrift für interkulturellen Fremdsprachenunterricht 11, H. 3, 1-21; online unter: http://tujournals.ulb. tu-darmstadt.de/index.php/zif/article/view/396/384 [Stand: 07.04.2017].

Rodríguez Pérez, Nieves / Heinsch, Bárbara (2013): Interculturalidad: ¿Quo vadis? Desde la aparición de la Interkulturelle Germanistik hasta la normativa europea vigente con especial énfasis en el contexto español. In: Futhark 8, 223-241.

Vollmer, Helmut Johannes (2010): Kompetenzforschung in den Fremdsprachendidaktiken - ein Überblick. In: Aguado, Karin / Schramm, Karen / Vollmer, Helmut Johannes (Hgg.): Fremdsprachliches Handeln beobachten, messen, evaluieren. Neue methodische Ansätze der Kompetenzforschung und der Videographie. Frankfurt a.M.: Lang, 29-64.

Weidemann, Arne / Nothnagel, Steffi (2010): Akteure. In: Weidemann, Arne / Straub, Jürgen / Nothnagel, Steffi (Hgg.): Wie lehrt man interkulturelle Kompetenz? Theorien, Methoden und Praxis in der Hochschulausbildung. Ein Handbuch. Bielefeld: Transcript, 123-162.

Wierlacher, Alois (2003): Interkulturalität. In: Wierlacher, Alois / Bogner, Andrea (Hgg.): Handbuch interkulturelle Germanistik. Stuttgart/Weimar: Metzler, 257-264.

Zarate, Geneviève (2003): Identities and plurilingualism: preconditions for the recognition of intercultural competences. In: Byram, Michael (Hg.): Intercultural Competence. Strasbourg: Council of Europe, 84-117; online unter: https://rm.coe.int/16806ad2dd [Stand: 09.07.2018].

Zarate, Geneviève (2010): Lévaluation des compétences culturelles et interculturelles à travers le débat des institutions européennes. In: Alao, George / Lecocq, Heba / Yun-Roger, Soyoung / Szende, Thomas (Hgg.): Implicites, stéréotypes, imaginaires: La composante culturelle en langue étrangère. Paris: Archives Contemporaines Editions, 7-17.

Dr. phil. Barbara Heinsch

Universität Oviedo, Institut für englische, französische und deutsche Philologie

heinschbarbara@uniovi.es 